\title{
Heinrich Wefelscheid Zum Siebzigsten Geburtstag
}

\author{
Joachim Bauer
}

\begin{abstract}
Zusammenfassung. Am 16. April 2011 vollendet der Mitbegründer und langjährige Geschäftsführende Herausgeber dieser Zeitschrift, Herr Prof. Dr. Dr. h.c. Heinrich Wefelscheid, sein siebzigstes Lebensjahr. Mit diesem runden Geburtstag wird er sich aus der Schriftleitung zurückziehen. Den Posten des Geschäftsführenden Herausgebers hatte er schon im vergangenen November an seinen Nachfolger übergeben. Die Zeitschrift ,,Resultate der Mathematik" -inzwischen umgetauft in ,,Results of Mathematics "ist eine Gründung der beiden Duisburger Mathematiker Hans Joachim Arnold und Heinrich Wefelscheid.
\end{abstract}

Es war um die Mitte der siebziger Jahre: Die sieben fetten Jahre der Universitätsgründungen, Stellenvermehrung und Mittelverteilung gingen erkennbar zu Ende, aber die sieben mageren Jahre der Stellenstreichung, k.w.-Vermerke, Mittelkürzungen und „Qualitätspaktschlüsse“ - die Politik ist im Erfinden von Euphemismen zur Mangelverwaltung ungemein phantasievoll-hatten noch nicht begonnen. Noch gab es ein wenig zu verteilen. Da trafen sich die beiden Duisburger Kollegen Arnold und Wefelscheid nach ihren Vorlesungen zum regelmäßigen Spaziergang. Bei dieser Gelegenheit bemerkte Herr Arnold, er sehe die Gelegenheit, Geld zur Gründung einer mathematischen Zeitschrift zu aquirieren. Herr Wefelscheid, der durch seinen Thales-Verlag in dieser Branche erfahren war, kalkulierte die für eine Anschubfinanzierung erforderlichen Mittel, und mit solchen Orientierungsdaten gerüstet, zog man zu Verhandlungen mit dem Ministerium nach Düsseldorf. Der für Duisburg zuständige Referent, Ministerialrat Schultheis, zeigte sich sehr entgegenkommend, und das nötige Geld wurde bewilligt. Auch die Verhandlungen mit dem Chef des Birkhäuser-Verlags, Herrn Einsele, einem Schwiegersohn des alten Herrn Birkhäuser, gestalteten sich erfreulich dank der Unterstützung durch 
Walter Benz, Helmut Karzel und Emanuel Sperner. Nach der Gründung der Zeitschrift im Jahr 1977 erschien 1978 der erste Band der ,,Resultate“. Zuerst gab es pro Jahr zwei, später vier Hefte; nach dem Übergang zum Offset-Druck konnte der Umfang verdoppelt werden auf insgesamt 800 Seiten eines Jahrgangs.

Ich habe in der Biographie des Jubilars schon ein wenig vorgegriffen. Der guten Ordnung halber zurück zum Anfang:

Heinrich Wefelscheid wurde am 16.04.1941 in Pernitz geboren. Dieser Ort im Wienerwald war weit genug vom Ruhrgebiet entfernt, um im Jahr 41 vor Bombenangriffen Sicherheit zu bieten. Die ersten Volksschuljahre nach dem Krieg verbrachte er in Neuss und Düsseldorf und die ersten Gymnasialjahre in Bochum. Sein Vater war promovierter Biologe und Oberstudiendirektor. 1956 erwarb der Vater ein Haus in Essen-Bredeney, und Heinrich Wefelscheid wechselte auf das Goethe-Gymnasium in Essen. Es war die Schule, an der sein Vater bis zur kurz zuvor erfolgten Pensionierung unterrichtet hatte. Als der Klassenlehrer seinen neuen Schüler nicht nur im Vorübergehen en profil, sondern zum erstenmal vor der Klasse en face wahrnahm, entfuhr ihm spontan die logisch unvollständige Frage: ,,Sind Sie verwandt?“, und der Sekundaner Wefelscheid antwortete spontan ebenso unvollständig ,ja“, was für Heiterkeit sorgte. Beide hatten unausgesprochen das fehlende Hinterglied der gemeinten zweistelligen Relation mitgedacht. 1960 bestand er dort die Reifeprüfung und entschied sich für ein Studium des Lehramts an Gymnasien. Das Berufsbild war ihm ja aus der Familie vertraut. Als Fächerkombination wählte er Mathematik und Physik, als Studienort die ehrwürdige Georgia Augusta in Göttingen. C.L. Siegel war gerade emeritiert worden, so dass Herr Wefelscheid keine Gelegenheit mehr hatte, bei ihm eine Vorlesung zu hören. Das Anfängerkolleg in Analytischer Geometrie und Algebra hielt Kurt Reidemeister. Es war Reidemeisters letzte Vorlesung vor der Emeritierung.

Diese Vorlesung hieß an deutschen Universitäten in alter Zeit ,Analytische Geometrie“, dann ,Analytische Geometrie und Algebra“, danach ,Lineare Algebra“ und war unter fast vollständiger Zurückdrängung der Geometrie doppelt unterstrichen linear algebraisch geworden, eine Entwicklung, die Reidemeister contre cœur ging. Er bot daher mit seiner Vorlesung einen Gegenentwurf. Die Vorlesung ist von H. Zieschang ausgearbeitet und später in hektographierter Form herausgegeben worden. Sie enthält einen Abschnitt über Grundlagen der Geometrie und dort eine originelle Einführung der Streckenrechnung mittels des Höhenschnittpunktsatzes. Damit die Studenten aber auch die orthodoxe lineare Algebra lernten, empfahl Reidemeister ihnen das bekannte Spernersche Lehrbuch zur vorlesungsbegleitenden Lektüre. In den Übungen sorgten dann seine Assistenten Gerhard Burde und Heiner Zieschang — später Professoren in Frankfurt/Main und Bochum - dafür, dass dies auch gründlich geschah. Bemerkenswerterweise empfahl er die ältere Auflage dieses Buches von Schreier und Sperner, die er für besser hielt. 
Reidemeisters Vorlesung war für die durchschnittlich begabten Anfänger schwer verständlich. Seine originelle Gedankenführung lockte zahlreiche fortgeschrittene Hörer in das Anfängerkolleg, die besonders Reidemeisters hochpointierte Anmerkungen zum politischen Tagesgeschehen genossen. Kritische Bemerkungen zur Politik in einer Vorlesung sind im liberalen Staat für einen Professor ungefährlich. Während der NS-Diktatur hatten sie Reidemeisters Strafversetzung zur Folge gehabt, als eines ,,Deutschen Beamten, der dem Führer nicht folgen will."

Die Theoretische Mechanik hörte Herr Wefelscheid bei Friedrich Hund, der drei Jahre zuvor von Frankfurt/Main einen Ruf nach Göttingen angenommen hatte - sehr zum Gram eines Frankfurter Buchhändlers, der sich mit den Hundschen Lehrbüchern zur Theoretischen Physik, Band I, II, III die Lager gefüllt und sie nun auf Halde liegen hatte. ,In meinem Buch ist nichts schwer", pflegte Hund zu sagen. Wenn ein Student ihn um Aufklärung einer unverstandenen Stelle bat, entließ er manchmal mit diesen Worten den unglücklichen Frager aus seiner Sprechstunde, ihn auf drastische Weise darauf aufmerksam machend, dass wissenschafltiches Studium kein Nürnberger Trichter ist, sondern eigenes Gehirnschmalz erfordert. Seiner oft bissigen Bemerkungen wegen wirkte er einschüchternd auf seine Hörer und war als, scharfer Hund" gefürchtet.

Nach fünf Semestern in Göttingen wechselten Heinrich Wefelscheid und ein Studienfreund die Universität. Herr Wefelscheid ging nach Heidelberg, der Studienfreund nach Hamburg. Beide verabredeten, einander von ihren Studien zu berichten. Danach wollte man entscheiden, wo es interessanter sei, und dort gemeinsam weiterstudieren.

Nach zwei Semestern in Heidelberg fiel die Entscheidung für Hamburg. Dort hatten die Mathematiker ein Tutorenprogramm eingerichtet. Herr Wefelscheid erhielt eine dieser (bezahlten) Tutorenstellen und kam in das Geometrieseminar von Sperner, Karzel und Joussen. Dieses Seminar hatte etwa 40 Teilnehmer. Es wurde ein Text zugrunde gelegt, und jeder war verpflichtet, in jeder Stunde vorbereitet zu sein. Herr Karzel forderte zu Beginn einer Sitzung einen beliebigen Teilnehmer zum Vortrag auf. Erwies dieser sich als unzureichend vorbereitet, so wandte er sich an einen der Tutoren, die gerade zu dem Zweck anwesend waren, damit das Seminar ohne Stocken seinen Fortgang nehmen konnte. Nur so ließ sich ein mathematisches Seminar mit hoher Teilnehmerzahl organisatorisch bewältigen.

Helmut Karzel begann damals als junger Professor in Hamburg, seine ersten Schüler und späteren Mitarbeiter heranzuziehen. Er hatte eine Arbeitsgemeinschaft für Staatsexamenskandidaten eingerichtet, und Heinrich Wefelscheid gehörte zur ersten Gruppe von Studenten, die bei ihm das Staatsexamen ablegten. Im darauffolgenden Jahr wurde er mit einer Dissertation über Vervollständigung topologisch-algebraischer Strukturen promoviert. Helmut Karzel war sein Doktorvater. Er suchte nun für seinen frisch promovierten Schüler eine freie Assistentenstelle und wurde bei Erich Kähler fündig. 
Dieser hatte eine unbesetzte Assistentenstelle. Er lieh sie an Herrn Karzel aus als freischwebend unbefristete Leihgabe mit der Nebenbedingung der Rückforderung, falls er, Kähler, sie wieder benötigen sollte. Dieser Fall ist aber nicht eingetreten.

Im anschließenden Jahr bot sich für Herrn Wefelscheid die Gelegenheit zum Aufenthalt an der Staatsuniversität von Pennsylvania. Der Logiker Schütte hatte dort auf Einladung von Church eine Gastprofessur inne. Er besetzte eine seiner Folgestellen mit Herrn Wefelscheid. Zur selben Zeit las auch H. Hasse als Gastprofessor an dieser Universität, und Herr Wefelscheid konnte die interessante Hasse'sche Vorlesung über Klassenkörpertheorie hören. Nach einjährigem Amerikaaufenthalt kam Herr Wefelscheid Ende August 1968 wieder nach Deutschland zurück. Auf der DMV-Tagung in Linz, der Gemeinschaftstagung mit der Österreichischen Mathematischen Gesellschaft, traf er alle bekannten Mathematiker aus Hamburg wieder.

Herr Sperner hatte gerade mehrere Ratsstellen zur Disposition, und Herr Karzel, inzwischen als Ordinarius nach Hannover berufen, konnte Herrn Wefelscheid eine davon vermitteln. So kam er wieder nach Hamburg zurück. Der Ordnung halber legte er, neben seiner Tätigkeit an der Universität, noch die zweite Staatsprüfung für das Lehramt an Gymnasien ab. 1972 habilitierte er sich mit „Untersuchungen über Fastkörper und Fastbereiche“. Vom gleichen Jahr an war er Jahrverwalter der Mathematischen Gesellschaft in Hamburg. Dieses Amt übte er 4 Jahre lang aus bis zu seiner Berufung als Professor nach Duisburg 1976. Die Mathematische Gesellschaft in Hamburg ist die älteste mathematische Vereinigung. Seit 1881 verfügt sie über eine eigene Zeitschrift, die bis 1941 regelmäßig erschien und dann kriegsbedingt eingestellt wurde. Das erste Heft nach dem Krieg kam 1959 heraus, die weiteren folgten bis 1971 in unregelmässigen Abständen. Herr Wefelscheid ist Mitherausgeber dieser ,Mitteilungen der Mathematischen Gesellschaft in Hamburg“ und gehörte der Schriftleitung an. Als Jahrverwalter sorgte er dafür, dass diese Zeitschrift von da ab wieder regelmäßig erschien in der ansprechenden äußeren Form, wie wir sie heute kennen. Zusammen mit einem Schulfreund hatte Herr Wefelscheid den Thales-Verlag gegründet, der je nach Bedarf aktiviert und wieder inaktiviert werden konnte. Im Thales-Verlag erschienen unter seiner Mitarbeit die Gesammelten Werke von Wilhelm Blaschke sowie die Monographie ,,Theorie der Fastkörper" von Heinz Wähling. Er war geschäftsführender Herausgeber der gesammelten Werke von Edmund Landau und, zusammen mit S.D. Chatterji, Herausgeber ausgewählter Werke von G.C. Young und W.H. Young.

Eine ehemalige Primanerin seines Vaters war Künstlerin geworden, hatte ,,eine gute Partie“ gemacht und verfügte nach dem Tod ihres Gatten über einiges Vermögen. Herr Wefelscheid, der gut mir ihr befreundet war, konnte Sie von der Idee einer wissenschaftsunterstützenden Stiftung überzeugen. Seinem Rat folgend, vermachte Sie mit ihrem Testament ihre Mittel einer Stiftung für Kunst, Kultur und Wissenschaft, die Herr Wefelscheid daraufhin 
gründete, und deren Vorstandsvorsitzender er ist. Mit der Hilfe dieser ,,Dierks von Zweck-Stiftung " konnten zahlreiche Tagungen finanziell unterstützt, und ausländischen Wissenschaftlern die Teilnahme an Tagungen durch Zuwendungen zu den Aufenthaltskosten ermöglicht werden. Insbesondere jüngere und finanziell schlechter gestellte Mathematiker aus Ost- und Südost-Europa, sowie aus China, ermöglichte die Dierks von Zweck-Stiftung so wissenschaftliche Forschungsaufenthalte. Aber auch die Förderung kultureller Aktivitäten gehört zu den Leistungen der Stiftung.

Im Jahr 2002 verlieh die Universität Sofia Herrn Wefelscheid die Ehrendoktorwürde. Herrn Wefelscheids mathematische Interessen liegen auf den Gebieten Algebra und Geometrie. Hier widmet er sich dem Studium der topologisch-algebraischen Strukturen, insbesondere den topologischen Fastkörpern, den topologischen Dicksonschen Fastkörpern und den topologischen Fastbereichen, zu deren Theorie er wichtige Resultate beigetragen hat, ferner der Theorie der Hyperbelstrukturen sowie der $K$-Loops. Zusammen mit W. Kerby untersuchte er die Beziehungen zwischen scharf zweifach transitiven Gruppen und Fastbereichen sowie scharf dreifach transitiven Gruppen und KT-Feldern.

Wir kennen aus der Geschichte der Mathematik viele Beispiele für abstrakte, augenscheinlich extrem anwendungsferne Theorien, die überraschend Anwendungen in den Realwissenschaften erhalten, für die sie gar nicht konzipiert waren. Ein solches Beispiel ist auch die von Heinrich Wefelscheid und William Kerby eingeführte $K$-Loop.

Herr Wefelscheid war bei der Lektüre eines Artikels des Physikers A.A. Ungar aufgefallen, dass K-Loops mit guter Wirkung zur Darstellung der Geschwindigkeitsaddition in der speziellen Relativitätstheorie eingesetzt werden können.

Zunächst seien die oben schlagwortartig gestreiften Begriffe kurz referiert. Aus dem Körperbegriff entsteht der Begriff des Fastkörpers durch Verzicht auf eines der beiden Distributivgesetze als algebraische Struktur $(K,+, \cdot)$, so dass $(K,+)$ eine (abelsche) Gruppe, $\left(K^{*}, \cdot\right)$ eine Gruppe ist und zusätzlich $0 \cdot a=0$ für alle $a \in K$ gilt, was man wegen der fehlenden Rechtsdistributivität fordern muss.

Dicksonsche Fastkörper (Dickson 1905) waren die ersten Fastkörperbeispiele. Man erhält sie aus gewöhnlichen (endlichen) Körpern, indem man anstelle der gewöhnlichen Multiplikation auf $K$ eine neue Multikation $\circ$ mittels einer sogenannten ,,Koppelungsabbildung " $\kappa$ einführt.

$a \circ x=a \cdot \kappa_{a}(x)$ für alle $a \in K^{*}$ und $0 \cdot x=0$. Hierbei ist

$$
\kappa:\left\{\begin{array}{l}
K^{*} \rightarrow \text { Aut } K \\
a \rightarrow \kappa_{a}
\end{array}\right.
$$

ein Homomorphismus von $K^{*}$ in die Automorphismengruppe von $K$ bzw. von $(K,+)$. 
Verzichtet man noch auf das Assoziativgesetz in $(K,+)$ und verlangt nur, dass in der Gleichung $x+y=z$ je zwei Elemente eindeutig das dritte bestimmen (Axiom L1) sowie die Existenz eines neutralen Elements 0 mit $0+a=a+0=a$ (Axiom L2), so gelangt man zur Struktur eines Fastbereichs. Hier ist das auf $(K,+)$ fehlende Assoziativgesetz zusätzlich durch das schwächere Axiom $K A$ zu ersetzen.

$K A: a+(b+x)=(a+b)+d_{a, b} \cdot x$ mit eindeutig bestimmtem, von $a$ und $b$ abhängigem $d_{a, b} \in K^{*}$.

Der Begriff des Fastbereichs wurde von Helmut Karzel eingeführt. Eine Struktur $(L,+)$, die $L 1$ und $L 2$ erfüllt, nennt man eine Loop. Erfüllt sie noch die weiteren Axiome

L3: zu $a, b$ gibt es einen Automorphismus $\delta_{a, b} \in \operatorname{Aut}(L,+)$ mit $a+(b+x)=$ $(a+b)+\delta_{a, b}(x)$ für alle $x \in L$,

L4: $(-a)+(-b)=-(a+b)$,

$L 5: \delta_{a, b}=\delta_{a, b+a}$,

so nannten sie Heinrich Wefelscheid und William Kerby, die diesen Begriff eingeführt haben, zu Ehren ihres Lehrers Helmut Karzel eine $K$-Loop.

Nun zum Zusammenhang dieser Struktur mit der speziellen Relativitätstheorie. Bewegt sich ein Beobachter II mit der konstanten Geschwindigkeit $\vec{u}$ in Bezug auf einen Beobachter I und ein Objekt III mit der konstanten Geschwindigkeit $\vec{v}$ in Bezug auf den Beobachter II, so bezeichnen wir mit $\vec{u} \oplus \vec{v}$ die Geschwindigkeit, die I misst, wenn er III beobachtet, (wobei II $\vec{v}$ misst, wenn er III beobachtet). In der Newton'schen Mechanik ist $\vec{u} \oplus \vec{v}$ die gewöhnliche Summe der gewöhnlichen Vektorrechnung. Nicht so in der Relativitätstheorie. Unkompliziert ist die Addition noch, wenn beide Geschwindigkeiten linear abhängig sind. Dann können die Koordinatensysteme so gewählt werden, dass beide sich auf einer Geraden bewegen und die relativistische Rechnung ergibt $u \oplus v=\frac{u+v}{1+u \cdot v}$ (der Betrag der Lichtgeschwindigkeit sei 1 gewählt) für die relativistisch erlaubten Geschwindigkeiten $|u|<1$ und $|v|<1$. Verwickelter gestaltet sich die Rechnung, wenn $\vec{u}$ und $\vec{v}$ linear unabhängig sind. Die Geschwindigkeitsaddition erweist sich dann als nichtkommutativ und nichtassoziativ. Im Formelapparat der relativistischen Rechnung taucht eine Drehmatrix auf, die sogenannte Thomas-Rotation $\operatorname{Tom}_{\vec{u}, \vec{v}}$, die von $\vec{u}, \vec{v}$ abhängt und einen Automorphismus des dreidimensionalen Raumes der physikalisch zulässigen Geschwindigkeiten beschreibt. A.A. Ungar berechnete, dass mit dem Matrizenprodukt $\operatorname{Tom}_{\vec{u}, \vec{v}} \vec{x}=\delta_{\vec{u}, \vec{v}}(\vec{x})$ die Abweichung der Geschwindigkeitsaddition von der Kommutativität durch

$$
\vec{u} \oplus \vec{v}=\delta_{\vec{u}, \vec{v}}(\vec{v} \oplus \vec{u})
$$

und von der Assoziativität durch

$$
\vec{u} \oplus(\vec{v} \oplus \vec{x})=(\vec{u} \oplus \vec{v}) \oplus \delta_{\vec{u}, \vec{v}}(\vec{x})
$$


beschrieben wird. Herr Wefelscheid bemerkte, dass $\delta_{\vec{u}, \vec{v}}$ ein Automorphismus ist und daher die physikalisch zulässigen Geschwindigkeiten mit der relativistischen Geschwindigkeitsaddition eine $K$-Loop bilden. Mit seiner Entdeckung hat Herr Wefelscheid den Anstoß zu zahlreichen Arbeiten auf diesem Feld gegeben.

Ein weiterer Begriff ist eng mit dem Namen Heinrich Wefelscheid verbunden. Der Salon, Pariser Platz 6a, in Berlin. In allerbester Lage ist es Herrn Wefelscheid gelungen, Miteigentümer eines für Empfänge und Veranstaltungen heißbegehrten Salons zu werden. Großzügig stellt er diese Kleinod unentgeltlich als Treffpunkt und Veranstaltungsort für nationale und internationale wissenschaftliche Tagungen zur Verfügung. Die internationale Mathematische Union, die Euler-Vorlesung, DMV-Treffen oder die Arbeitswochen über Geometrie und Algebra sind hier unter vielen anderen aufs Beste empfangen und versorgt worden. Das prall gefüllte Gästebuch bezeugt die große Anzahl internationaler Wissenschaftler die das Brandenburger Tor und das Reichstagsgebäude aus nächster Nähe bewundert haben und die großzügige Gastfreundschaft von Herrn Wefelscheid genossen haben.

Die Kollegen der Fakultät für Mathematik in Duisburg wünschen Herrn Wefelscheid noch viele Lebensjahre bei Gesundheit und Wohlergehen.

Da Langlebigkeit ein Attribut seiner Familie ist, hat dieser Wunsch berechtigte Aussicht auf Erfüllung. Er möge diese Jahre zubringen bei unbeschwert heiterer Gemütsverfassung und mit einem allzeit gefüllten Glas Champagner.

Joachim Bauer

Fakultät für Mathematik

Universität Duisburg-Essen

Forsthausweg 2

47057 Duisburg

Germany 